\begin{tabular}{|c|l|}
\hline Title & Role of the d-d interaction in the antiferromagnetic phase of lambdz(BEDT-STF)(2)FeCI4 \\
\hline Author(s) & $\begin{array}{l}\text { Minamidate, Takaaki; Shindo, Hironori; I hara, Y oshihiko; Kawamoto, A tsushi; Matsunaga, Noriaki; Nomura, } \\
\text { Kazushige }\end{array}$ \\
\hline Citation & $\begin{array}{l}\text { Physical Review B, 97(10), 104404 } \\
\text { https://doi.org/10.1103/PhysRevB.97.104404 }\end{array}$ \\
\hline Issue Date & 2018-03-05 \\
\hline Doc URL & http://hdl.handle.net/2115/70127 \\
\hline Rights & (C)2018A merican Physical Society \\
\hline Type & article \\
\hline File Information & PhysRevB.97.104404.pdf \\
\hline
\end{tabular}

Instructions for use 


\title{
Role of the $d-d$ interaction in the antiferromagnetic phase of $\lambda$-(BEDT-STF) $)_{2} \mathrm{FeCl}_{4}$
}

\author{
Takaaki Minamidate, Hironori Shindo, Yoshihiko Ihara, Atsushi Kawamoto, Noriaki Matsunaga, and Kazushige Nomura \\ Department of Physics, Hokkaido University, Sapporo 060-0810, Japan
}

(Received 4 April 2017; revised manuscript received 9 January 2018; published 5 March 2018)

\begin{abstract}
Magnetic susceptibility and proton nuclear magnetic resonance $\left({ }^{1} \mathrm{H}-\mathrm{NMR}\right)$ measurements were performed for the quasi-two-dimensional $\pi-d$ interacting system $\lambda$-(BEDT-STF $)_{2} \mathrm{FeCl}_{4}$ at ambient pressure. Magnetic susceptibility arising from the $3 d$ spins of the $\mathrm{FeCl}_{4}$ anion show an anisotropy at low temperature and its temperature dependence for the external field parallel to the $c$ axis is described as a broad peak structure at $8 \mathrm{~K}$. A sharp peak in the temperature dependence of $T_{1}^{-1}$ associated with the antiferromagnetic (AF) transition is observed at $T_{\mathrm{AF}}=16 \mathrm{~K}$, together with the drastic splitting of the NMR spectrum below $T_{\mathrm{AF}}$. The relation between the static susceptibility and the splitting of the NMR shift suggests the existence of the relatively strong $d$ - $d$ AF interaction. These results can be explained by the model considering the AF-coupled $d$-spin system in the AF long-range-ordered $\pi$-spin system. We find that the AF phases in $\lambda$-type salts can be universally explained by this model.
\end{abstract}

DOI: 10.1103/PhysRevB.97.104404

\section{INTRODUCTION}

The study of the $\pi$ - $d$ interacting system has been one of the most intensely investigated topics in the field of strongly correlated electron systems. Low-dimensional organic conductors are important examples of the $\pi-d$ system and, in particular, the quasi-two-dimensional organic conductor $\lambda$-(BETS) ${ }_{2} \mathrm{FeCl}_{4}$ [BETS $=$ bis(ethylenedithio)tetraselenafulvalene] is well known as the first organic material to show a magnetic-fieldinduced superconductivity $[1,2]$, which is explained by the Jaccarino-Peter compensation mechanism, where the external magnetic field compensates the internal field of aligned $d$ spins through the $\pi-d$ interaction [3]. At zero field, $\lambda$-(BETS) ${ }_{2} \mathrm{FeCl}_{4}$ shows a metal-antiferromagnetic (AF) insulator transition at $T_{\mathrm{AF}}=8.3 \mathrm{~K}$, and the AF phase is destabilized by a magnetic field of approximately $10 \mathrm{~T}$. Below $1 \mathrm{~K}$, a superconducting phase appears above $17 \mathrm{~T}$ when the magnetic field is applied parallel to the conducting layers. As the $\mathrm{Fe}^{3+}$ ion is in the $S=$ $5 / 2$ high-spin state and the magnetization shows anisotropy below $T_{\mathrm{AF}}$, it has been believed so far that the metal-AF transition is caused by the AF ordering of the Fe moments [4-6].

However, in a recent study, Akiba et al. found the broad hump structure in the temperature dependence of the specific heat and explained this structure by invoking the Schottkytype behavior due to the freedom of the rotation of the $3 d$-spin system in the internal field, which is induced by the surrounding AF-ordered $\pi$-spin system [7]. From the fitting of the Schottky curve with $S=5 / 2$, they estimated the internal field to be $H_{\text {int }} \sim 4 \mathrm{~T}$. Since they proposed that the $\pi$-spin system becomes an AF long-range-ordered state at $T_{\mathrm{AF}}$, in this paper, we hereafter refer to this model as the " $\pi$-ordering model." They also focused on the broad shoulder-like anomaly of the spin susceptibility below $T_{\mathrm{AF}}$ and explained this anomaly by using $\pi$-ordering model [8]. They proposed that, since $H_{\text {int }}$ alternately changes its direction at each Fe site, the local magnetizations of the $3 d$ spins, which obey the simple Brillouin function, are canceled out at the region, where the Brillouin function saturates. Therefore, the susceptibility decreases at low temperature. Furthermore, according to the torque measurement, the tilt angle $\theta$ between the easy axis and the $c$ axis is estimated as $30^{\circ}$ in the $c-b^{*}$ plane [9]. Moreover, according to the sextet Fe Mössbauer signals, $H_{\text {int }} \sim 4 \mathrm{~T}[10]$. These values obtained from magnetic torque and Mössbauer measurements are in good agreement with those estimated from the specific heat and static susceptibility using the $\pi$-ordering model $[7,8]$.

In a previous study, we found that $\lambda$-(BEDT-STF $)_{2}$ $\mathrm{GaCl}_{4}$, where BEDT-STF $=$ bis(ethylenedithio)diselenadithiafulvalene (hereafter, we abbreviate BEDT-STF as STF), shows a superconducting phase above $1.22 \mathrm{GPa}$, which means that $\lambda$-(STF $)_{2} \mathrm{GaCl}_{4}$ can be located at a lower-pressure region than $\lambda$-(BETS) ${ }_{2} \mathrm{GaCl}_{4}$ [11]. Furthermore, we found that the $\lambda$-(STF $)_{2} \mathrm{GaCl}_{4}$ does not show an AF phase at ambient pressure and the temperature dependence of the static spin susceptibility can be well explained by the AF Heisenberg model with a regular triangular lattice, with the $\mathrm{AF}$ exchange interaction in the $\pi$-electron system, $J / k_{\mathrm{B}}=-165 \mathrm{~K}$. Although it shows a very large $J$, this salt does not show AF ordering, at least down to $2 \mathrm{~K}$. It seems to be caused by the spin frustration due to the geometry of the AF spin interaction.

To elucidate the mechanism of the AF transition in the $\lambda$-FeCl${ }_{4}$ families, we measured the magnetic susceptibility and proton nuclear magnetic resonance $\left({ }^{1} \mathrm{H}-\mathrm{NMR}\right)$ at ambient pressure of $\lambda$-(STF $)_{2} \mathrm{FeCl}_{4}$, which is expected to be located in a lower-pressure region than $\lambda$-(BETS $)_{2} \mathrm{FeCl}_{4}$ in the phase diagram from the same analogy as that for the $\lambda-\mathrm{GaCl}_{4}$ salts. Herein, we report the results of the measurements and discuss the mechanism of the AF transition in the quasi-twodimensional $\pi-d$ interacting system $\lambda-\mathrm{FeCl}_{4}$ families.

\section{EXPERIMENTS}

Single crystals of $\lambda$-(STF $)_{2} \mathrm{FeCl}_{4}$ were synthesized by the standard electrochemical method. We used $\mathrm{x}$-ray diffraction to determine the lattice parameters of the systemto 


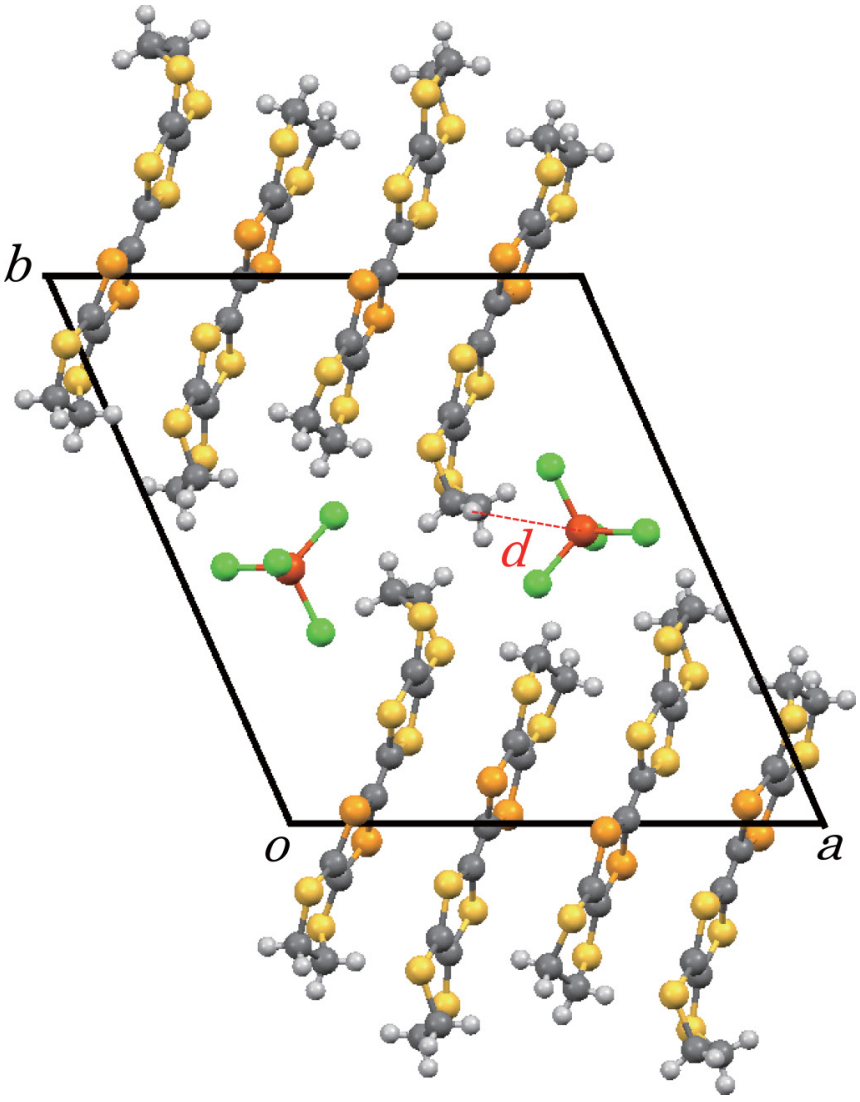

FIG. 1. Crystal structure of $\lambda$-(STF $)_{2} \mathrm{FeCl}_{4}$ projected along the $c$ axis. The red broken line denotes the shortest distance $d=3.439 \AA$ between the $\mathrm{Fe}$ atom and proton sites.

be $a=16.202(3) \AA, b=18.286(4) \AA, c=6.5645(16) \AA$, $\alpha=98.443(6)^{\circ}, \beta=96.799(7)^{\circ}$ and $\gamma=112.347(3)^{\circ}$, which are consistent with a previous report [12]. We obtained needle crystals with the long axis parallel to the $c$ axis. The crystal structure is shown in Fig. 1. The electronic resistivity measurement was done by using the dc four-probe method at ambient pressure. Electric leads of $\phi 10 \mu \mathrm{m}$ gold wires were attached to the sample by using a carbon paste. The current flows parallel to the $c$ axis. The static magnetic susceptibility measurement was performed by using a MPMS SQUID magnetometer (Quantum Design) for the aggregate of crystals aligned along the $c$ axis. The external magnetic field was applied parallel and perpendicular to the $c$ axis. Proton NMR measurements were performed down to $4.0 \mathrm{~K}$ under $3.117 \pm 0.15 \mathrm{~T}$ field corresponding to the resonance frequency of 132.5 MHz. Magnetic fields were applied perpendicular to the $c$ axis. Field-swept spectra were obtained by fast Fourier transformation (FFT) of the spin-echo signal with a $\pi / 2-\pi$ pulse sequence.

\section{RESULTS AND DISCUSSION}

Figure 2 shows the temperature dependence of the electronic resistivity of $\lambda$-(STF $)_{2} \mathrm{FeCl}_{4}$ at ambient pressure. In the whole temperature range, the resistivity increases with decreasing temperature. The system does not show any obvious anomaly down to $50 \mathrm{~K}$. This insulating behavior is consistent

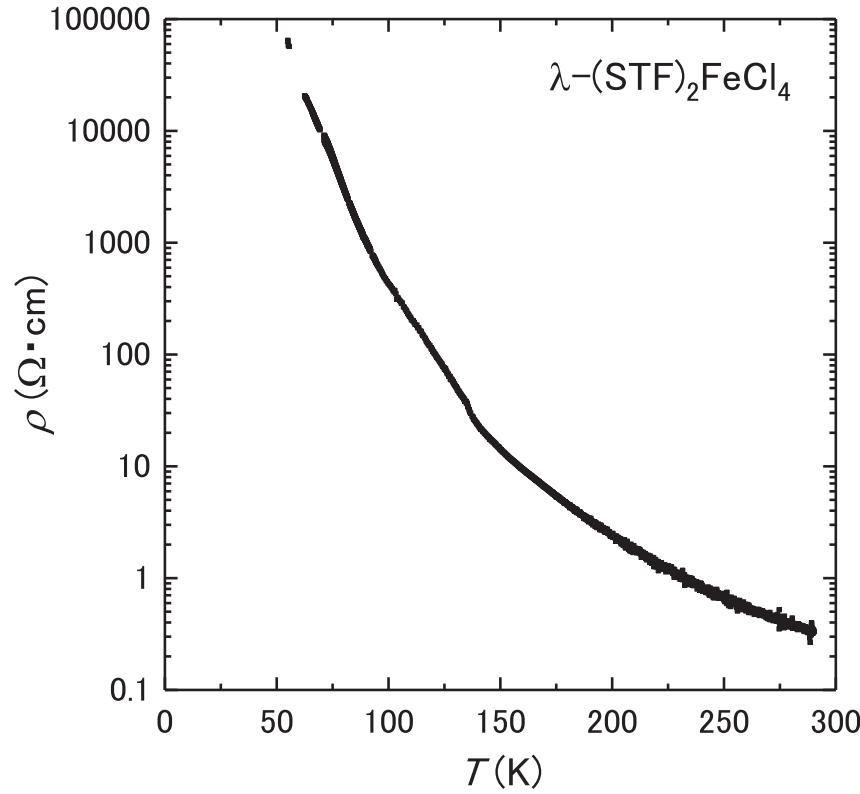

FIG. 2. Temperature dependence of the electronic resistivity of $\lambda$-(STF $)_{2} \mathrm{FeCl}_{4}$ at ambient pressure. The current flows parallel to the $c$ axis.

with that of $\lambda$-(STF $)_{2} \mathrm{GaCl}_{4}$ at ambient pressure $[11,12]$. Using the same analogy as that for $\mathrm{GaCl}_{4}$ salt, $\lambda$-(STF $)_{2} \mathrm{FeCl}_{4}$ can be located in the negative-pressure region of $\lambda$-(BETS ${ }_{2} \mathrm{FeCl}_{4}$ in the pressure phase diagram.

Figure 3 shows the temperature dependence magnetic susceptibility below $20 \mathrm{~K}$ for various fields. When a weak field is applied parallel to the $c$ axis, the magnetic susceptibility shows a broad peak around $8 \mathrm{~K}$ and gradual decreasing at low temperature. In the field range of $0.5-2 \mathrm{~T}$, the decreasing of the susceptibility is reduced gradually. Above $2 \mathrm{~T}$, the susceptibility increases with decreasing temperature down to $2 \mathrm{~K}$. On the contrary, with the weak perpendicular field to the $c$ axis, although the slope of the susceptibility becomes gentle around $8 \mathrm{~K}$, the susceptibility continues to increase in the low-temperature region. Similar to the case with parallel field, in the field range of 0.5-2 T, the susceptibility increases with increasing field. Above $3 \mathrm{~T}$, the anisotropy disappears completely.

Figure 4 shows the magnetic susceptibility observed under the external fields $B \| c$ (needle axis of the crystal) and $B \perp c$ at $0.1 \mathrm{~T}$. Above $20 \mathrm{~K}$, the magnetic susceptibility in both directions shows Curie-Weiss-type behavior for the $S=5 / 2$ high-spin state with the Weiss temperature $T_{\mathrm{W}}=-7.8 \mathrm{~K}$. The magnetic susceptibility for the field perpendicular to the $c$ direction continues to increase with decreasing temperature. On the contrary, in the field direction $B \| c$, the magnetic susceptibility shows a broad peak around $8 \mathrm{~K}$ and gradual decreasing at low temperature, which is reminiscent of the shoulder-like structure in the susceptibility behavior of $\lambda$-(BETS) ${ }_{2} \mathrm{FeCl}_{4}[8]$. As shown by the solid line in Fig. 4, we calculated the static susceptibility in the framework of the $\pi$-ordering model. This model takes into account the internal field on $d$-spin sites $\pm \boldsymbol{H}_{\text {int }}$ due to the alternately oriented $\pi-d$ exchange field from the AF-ordered $\pi$-spin system. In 


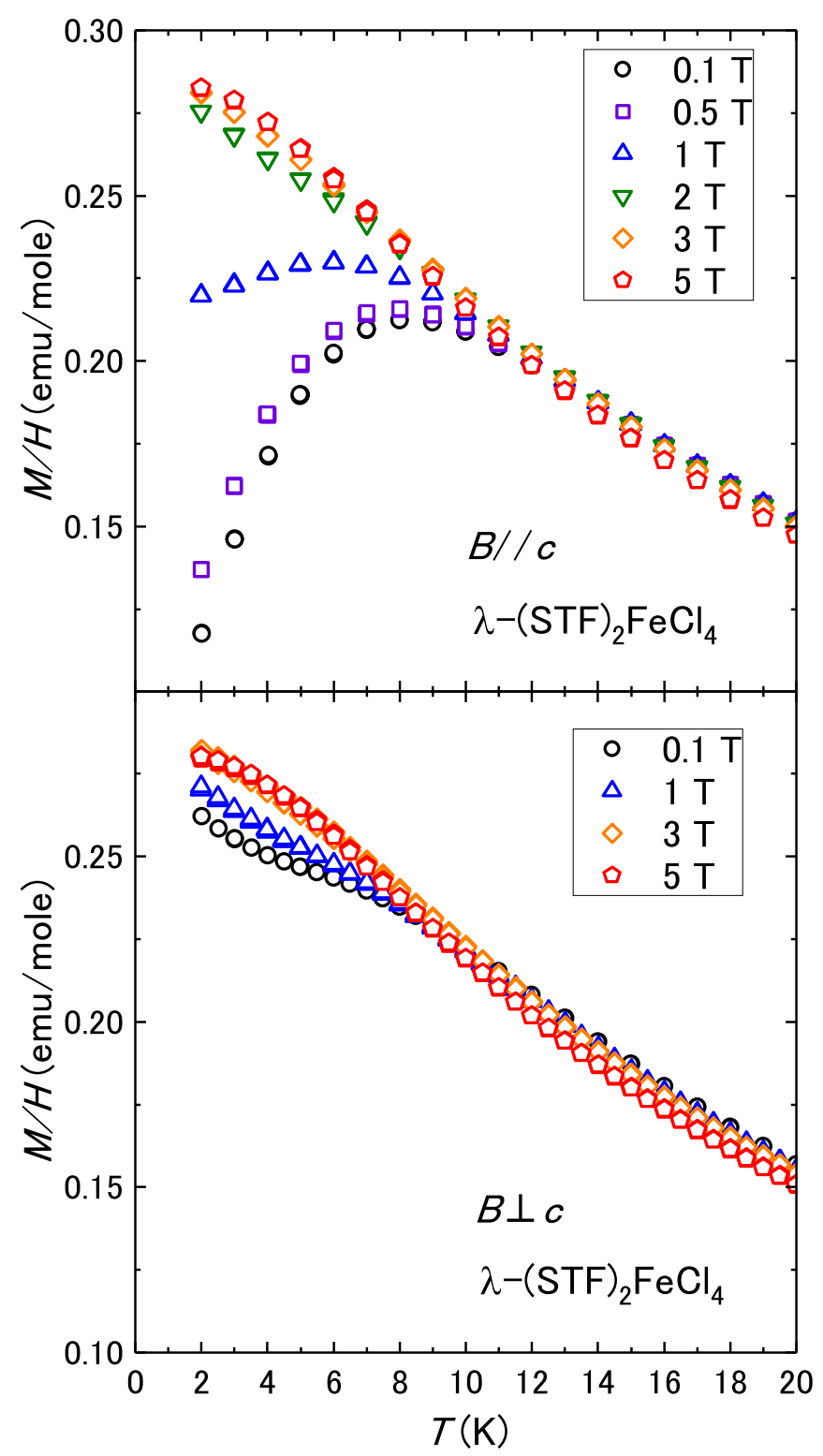

FIG. 3. Temperature dependence of static susceptibility in $\lambda$-(STF $)_{2} \mathrm{FeCl}_{4}$ for various magnetic fields. The measurements were made with the magnetic field parallel or perpendicular to the $c$ axis.

this model, the $d$-spin system generates the sublattice, and the magnetizations in each sublattice are described as

$$
M_{d}^{ \pm}=\frac{N}{2} g \mu_{\mathrm{B}} S_{d} B_{\mathrm{S}}\left(\frac{g \mu_{\mathrm{B}} \boldsymbol{S}_{d} \cdot \boldsymbol{H}_{\mathrm{eff}}^{ \pm}}{k_{\mathrm{B}} T}\right),
$$

where $N$ is the number of spins, $g$ is the $g$ value, $\mu_{\mathrm{B}}$ is the Bohr magneton, $\boldsymbol{S}_{d}$ is the $d$-spin moment, $B_{\mathrm{S}}$ is the Brillouin function, and the effective field $\boldsymbol{H}_{\text {eff }}^{ \pm}$is the sum of the external field $\boldsymbol{H}_{\text {ext }}$ and internal field $\pm \boldsymbol{H}_{\text {int }}$. Considering the tilt angle between the easy axis and the $c$ axis, the total magnetization component parallel to the external field can be obtained as

$$
M_{d}=M_{d}^{+} \cos (\alpha)+M_{d}^{-} \cos (\beta),
$$

where the $\alpha(\beta)$ is the angle between $\boldsymbol{H}_{\text {ext }}$ and $\boldsymbol{H}_{\text {eff }}^{+}\left(\boldsymbol{H}_{\text {eff }}^{-}\right)$. This model can explain the gradual rising of the anisotropy and decreasing of $\chi$ in the low-temperature region by the

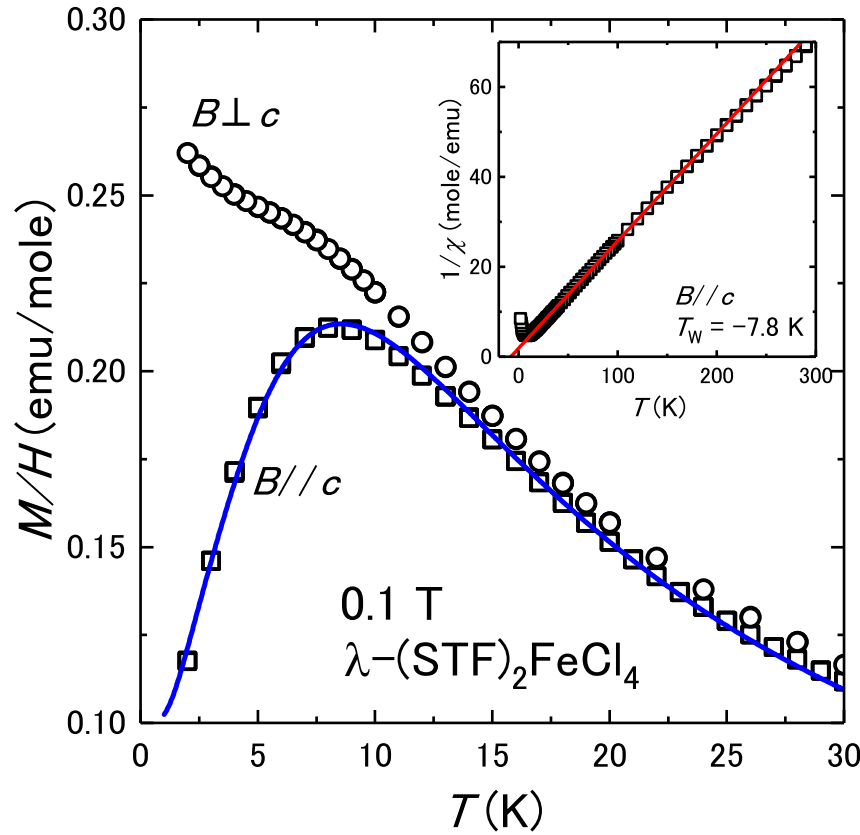

FIG. 4. Temperature dependence of static susceptibility in $\lambda$-(STF $)_{2} \mathrm{FeCl}_{4}$. Measurements for $B \| c$ (needle axis of crystal) and $B \perp c$ were performed for the same sample. The solid curve represents the magnetic susceptibility calculated on the basis of the AF-ordered $\pi$-spin model. The inset shows Curie-Weiss plots of the static susceptibility. The Weiss temperature $T_{\mathrm{W}}$ is estimated to be $-7.8 \mathrm{~K}$.

canceling out between the sublattice magnetizations. Using this model, the static susceptibility of $\lambda$-(STF $)_{2} \mathrm{FeCl}_{4}$ can be well explained with the parameters of $\theta=33^{\circ}$ and $\mu_{0} H_{\text {int }}=$ $4.7 \mathrm{~T}$. These values are close to those of $\lambda$-(BETS) ${ }_{2} \mathrm{FeCl}_{4}$. Thus, in the AF phase, the temperature dependence of the magnetic susceptibility has been well explained by the model considering the $d$-spin moments affected by the alternate oriented internal field.

Figure 5(a) shows the ${ }^{1} \mathrm{H}-\mathrm{NMR}$ spectra at $132.5 \mathrm{MHz}$ for a single crystal of $\lambda$-(STF) ${ }_{2} \mathrm{FeCl}_{4}$ at ambient pressure for various temperatures below $20 \mathrm{~K}$. A sharp peak is observed at $3.117 \mathrm{~T}$, corresponding to the signal from the free protons. Because the sharp peak at 3.117 $\mathrm{T}$ and the broad peak in the 3.150-3.165 T range, indicated by "*”, do not show temperature and field angular dependence, they can be regarded as signals from grease or contamination. At $20 \mathrm{~K}$, there are several signals from the proton sites of $\lambda$-(STF $)_{2} \mathrm{FeCl}_{4}$ in the 3.10-3.15 T range. As the temperature decreases from 20 to $16 \mathrm{~K}$, the spectrum gradually broadens and then shows a drastic splitting into multiple peaks observed below $16 \mathrm{~K}$. At the lowest temperature, the peaks, which are assigned to the 16 independent proton sites of the ethylene group, are broadened in the wide range of the field, 3.05-3.20 T, which is within the range expected from the maximum dipole field considering the shortest proton- $\mathrm{Fe}$ distance $d=3.439 \AA$ (red dashed line in Fig. 1). The local fields on each site are predominantly determined by the dipole magnetic field arising from the $3 d$ spin of the $\mathrm{Fe}^{3+}$ ions. Figure 5(b) shows the temperature dependence of $T_{1}^{-1}$ at a single spectrum peak marked as " $\nabla$." For all temperatures, the relaxation curve is described by the almost-single exponential. 

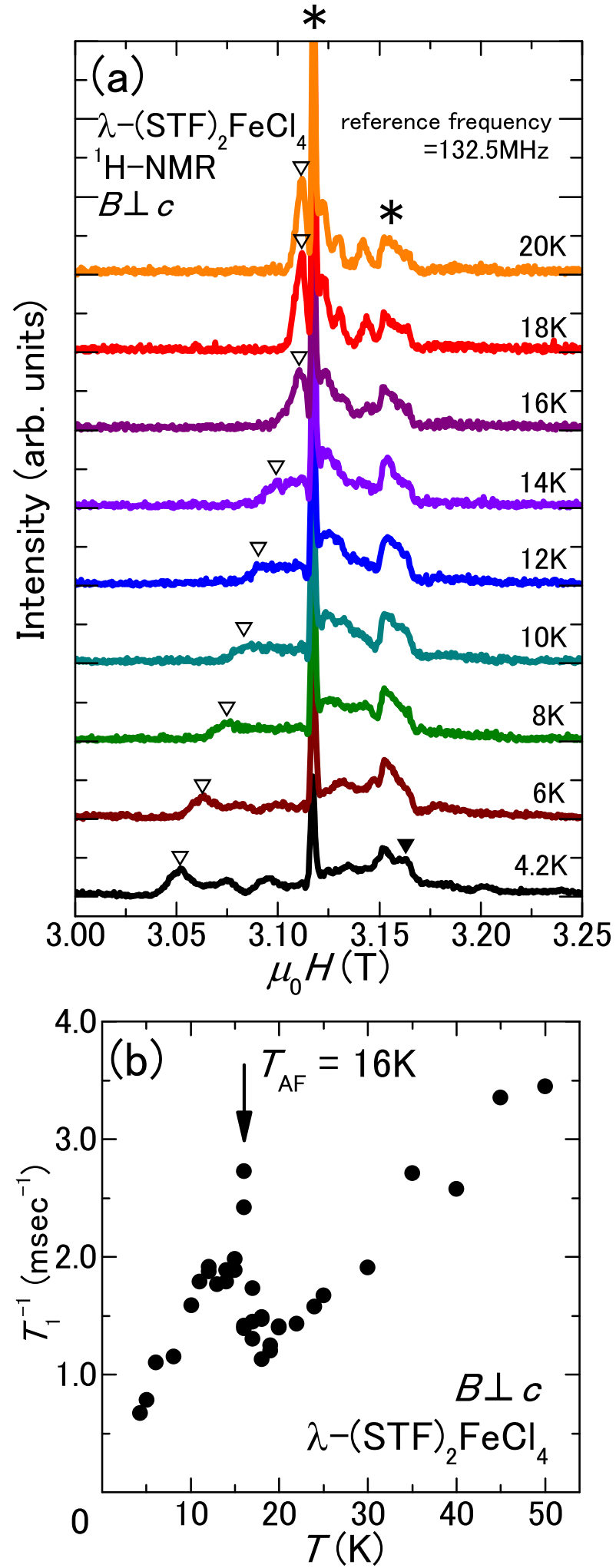

FIG. 5. (a) ${ }^{1} \mathrm{H}-\mathrm{NMR}$ field-swept spectra at $132.5 \mathrm{MHz}$ for a single crystal of $\lambda$-(STF $)_{2} \mathrm{FeCl}_{4}$ at ambient pressure. External magnetic field was applied perpendicular to the $c$ axis. Central sharp peaks at $3.117 \mathrm{~T}$ and broad peak in the range of 3.150-3.165 T (marked as “*”), which does not have temperature or field-rotation dependence, are considered to be signals from contamination or grease. (b) Temperature dependence of ${ }^{1} \mathrm{H}$-NMR $T_{1}^{-1}$ of $\lambda$-(STF $)_{2} \mathrm{FeCl}_{4}$ at ambient pressure. Magnetic fields were applied perpendicular to the $c$ axis.

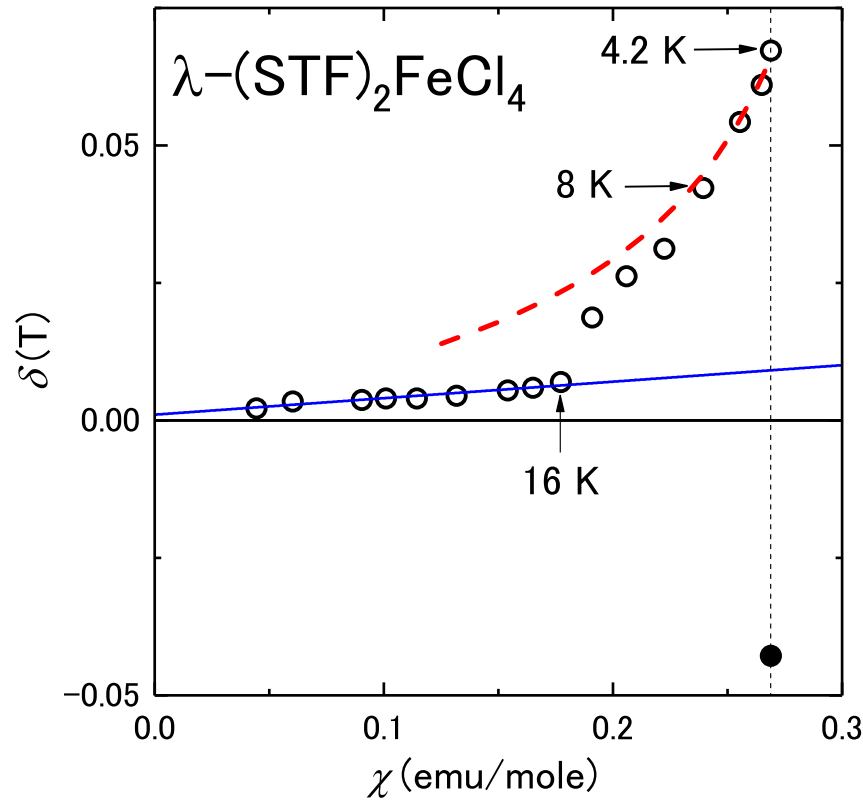

FIG. 6. $\delta-\chi$ plot of $\lambda$-(STF $)_{2} \mathrm{FeCl}_{4}$ for the peak marked by $\nabla$ and $\boldsymbol{\nabla}$ in Fig. 5(a). The red dashed line is the calculated $\delta-\chi$ relation using the model taking into account the $d-d$ interaction with the fitting parameters $\mu_{0} H_{\pi-d}=2 \mathrm{~T}$ and $\mathcal{A}=-2.9$.

The sharp peak observed at approximately $16 \mathrm{~K}$ is associated with the increase in low-frequency fluctuations due to the $\mathrm{AF}$ long-range ordering at $T_{\mathrm{AF}}$; the peak temperature corresponds to the drastic broadening temperature in the spectra. We determine $T_{\mathrm{AF}}=16 \mathrm{~K}$.

The observed magnetic susceptibility, shown in Fig. 4, does not exhibit any anomaly or anisotropy at $T_{\mathrm{AF}}$. This is because the Zeeman splitting energy of the $3 d$ spin $(S=5 / 2)$ under the internal magnetic field is negligibly smaller than the thermal energy $k_{\mathrm{B}} T_{\mathrm{AF}}$ because of the high $T_{\mathrm{AF}}$ in $\lambda$-(STF $)_{2} \mathrm{FeCl}_{4}$ and the $3 d$ spins behave as free spins around $T_{\mathrm{AF}}$. Therefore, the $\pi$-ordering model consistently explains the results of magnetic susceptibilities of the STF and BETS salts.

The $\delta-\chi$ relation is shown in Fig. 6, where $\chi$ is the static susceptibility at $3 \mathrm{~T}$ of the $c$-perpendicular field and $\delta$ is the NMR shift from the value of the free spin obtained from the peaks marked by $\nabla$ and $\boldsymbol{\nabla}$ in Fig. 5(a). $\delta$ is given by the $c$-perpendicular components of the local dipole field on the one proton site mainly arising from the magnetization of the nearest-neighbor Fe moment. On the other hand, $\chi$ corresponds to the total of the $c$-perpendicular components of Fe moments. Generally, in the paramagnetic state, the $\delta-\chi$ relation follows the formula of $\delta=K+\sigma=A \chi+\sigma$, where $K$ is the Knight shift, $A$ is the hyperfine coupling constant, and $\sigma$ is the chemical shift. Above $16 \mathrm{~K}$, the observed $\delta$ is proportional to $\chi$ with a negligibly small $\sigma$, as shown by the blue solid line in Fig. 6 . From the slope of the $\delta-\chi$ plot above $16 \mathrm{~K}$, we evaluate the hyperfine coupling constant to be $0.0056 \mathrm{~T} / \mu_{\mathrm{B}}$. Below $16 \mathrm{~K}, \delta$ deviates from the proportional relation and becomes drastically large. We find that the peak marked by $\boldsymbol{\nabla}$ is located at the position that is symmetrical to that of the peak marked by $\nabla$ in the spectrum at $4.2 \mathrm{~K}$, even though it is due to the extrinsic signal, the temperature dependence of the signal 
(a)
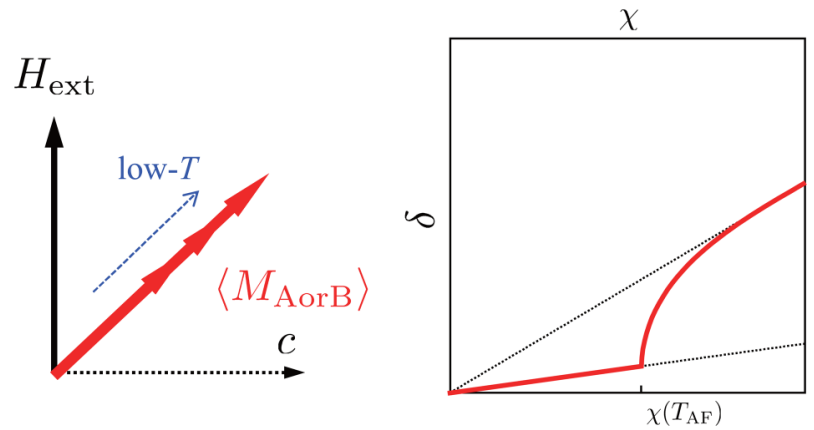

(b)

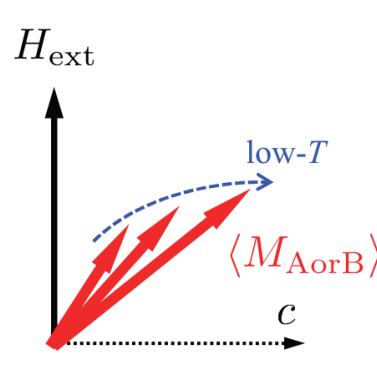

$\chi$

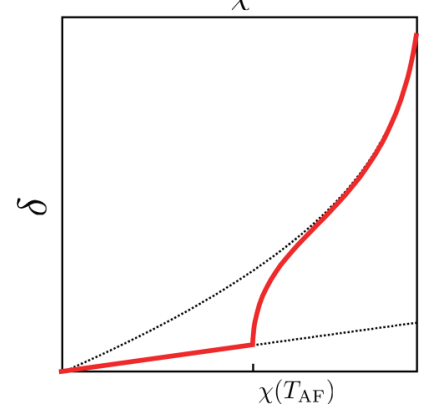

FIG. 7. Schematic drawing of evolution of magnetization on the $d$-spin site with changing temperature and expected temperature dependence of the $\delta-\chi$ relation for (a) original $\pi$-ordering model and (b) our model, which takes into account the $d$ - $d$ interaction.

marked by $\mathbf{\nabla}$ cannot be rigorously traced. This splitting of the NMR spectrum is caused by the occurrence of the $c$-parallel components of the $\mathrm{Fe}$ spin moments due to the $\mathrm{AF}$ transition.

We now discuss the $\delta-\chi$ relation in the $\pi$-ordering model. The original $\pi$-ordering model proposed by Akiba et al. considers the $\pi-d$ interaction as the source of the internal field on the $\mathrm{Fe}$ sites and ignores any other coupling [8]. In this case, as $\delta$ and $\chi$ are both proportional to the same Brillouin function, $\delta$ must be proportional to $\chi$ even though it has a different slope from the paramagnetic phase in the low-temperature region below $T_{\mathrm{AF}} / 2$, as shown in Fig. 7(a). It is because the $\pi-d$ exchange field almost saturates, as expected from the mean-field theory of AF. However, the observed $\delta-\chi$ relation in the AF phase is steeper than the proportional relation and $\delta$ continues to increase down to $4.2 \mathrm{~K}$ without any saturation. To explain this behavior, we must consider the AF exchange interaction in the $d$-spin system additionally to the simple $\pi$-ordering model. In the proposed model, as shown in Fig. 8, the $d$-spin moments in each sublattice are affected by $\boldsymbol{H}_{\text {eff, } \mathrm{A}(\mathrm{B})}=\boldsymbol{H}_{\text {ext }}+\boldsymbol{H}_{\pi-d, \mathrm{~A}(\mathrm{~B})}+\boldsymbol{H}_{d-d, \mathrm{~A}(\mathrm{~B})}$. (i) $\boldsymbol{H}_{\text {ext }}$ is the external field. In this model, for simplicity, it is assumed to be perpendicular to the $c$ axis. (ii) $\boldsymbol{H}_{\pi-d, \mathrm{~A}(\mathrm{~B})}$ is the internal field due to the $\mathrm{AF}$ exchange field from the either sublattice of the AF-ordered $\pi$-spin system. (iii) $\boldsymbol{H}_{d-d, \mathrm{~A}(\mathrm{~B})}$ is the internal field due to the exchange field from the opposite $d$-spin sublattice and is described as $\boldsymbol{H}_{d-d, \mathrm{~A}(\mathrm{~B})}=\mathcal{A}\left\langle\boldsymbol{M}_{\mathrm{B}(\mathrm{A})}\right\rangle$, where $\mathcal{A}$ denotes the strength of the $\mathrm{AF}$ interaction $\mathcal{A}<0$. As mentioned above, since the magnetizations of each sublattice in the AF-ordered $\pi$-spin system would nearly saturate, $\boldsymbol{H}_{\pi-d, \mathrm{~A}(\mathrm{~B})}$ would be constant below $T_{\mathrm{AF}}=8 \mathrm{~K}$. In contrast, as the temperature decreases, $\boldsymbol{H}_{d-d, \mathrm{~A}(\mathrm{~B})}$ grows at low temperature

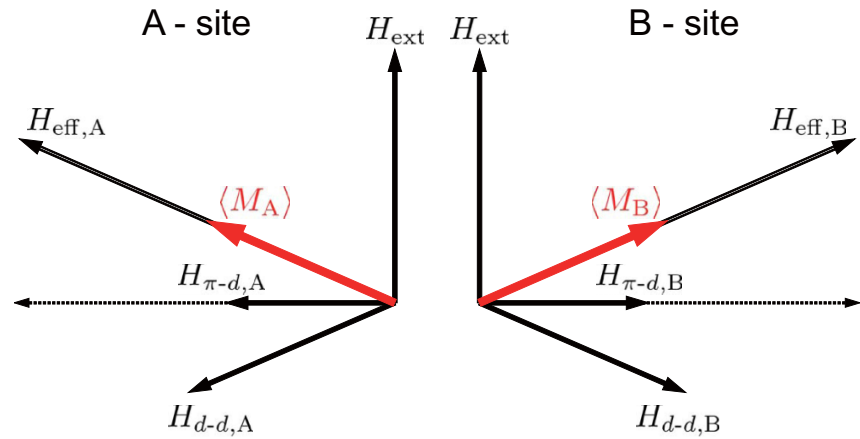

FIG. 8. Schematic of the magnetic fields and magnetization on each sublattice of the $d$-spin system. $\boldsymbol{H}_{\text {ext }}$ is the external field, $\boldsymbol{H}_{\pi-d}$ is the exchange field due to the ordered $\pi$-spin system, and $\boldsymbol{H}_{d-d, \mathrm{~A}(\mathrm{~B})}$ is the exchange field due to the opposite sublattice of $d$-spin system, $\boldsymbol{H}_{\mathrm{eff}, \mathrm{A}(\mathrm{B})}=\boldsymbol{H}_{\mathrm{ext}}+\boldsymbol{H}_{\pi-d, \mathrm{~A}(\mathrm{~B})}+\boldsymbol{H}_{d-d, \mathrm{~A}(\mathrm{~B})}$ is the effective field, and $\left\langle\boldsymbol{M}_{\mathrm{A}(\mathrm{B})}\right\rangle$ is the magnetization of $d$-spin sublattice A (B).

with increasing $\left\langle\boldsymbol{M}_{\mathrm{B}(\mathrm{A})}\right\rangle$, which obeys the Brillouin function (1). The changing ratio between $\boldsymbol{H}_{\pi-d}$ and $\boldsymbol{H}_{d-d}$ gives rise to the rotation of the $d$-spin moment with decreasing temperature, as shown in Fig. 7(b). In this case, we can describe the steep increase of the $\delta-\chi$ relation below $8 \mathrm{~K} . \chi$ is calculated as $\left|\left\langle\boldsymbol{M}_{\mathrm{A}}\right\rangle+\left\langle\boldsymbol{M}_{\mathrm{B}}\right\rangle\right| / \boldsymbol{H}_{\mathrm{ext}}$ and the splitting in $\delta$ is proportional to the amplitude of $\left\langle\boldsymbol{M}_{\mathrm{A}}\right\rangle-\left\langle\boldsymbol{M}_{\mathrm{B}}\right\rangle$. Accordingly, the points of the arrow in Fig. 7(b) describe the $\delta-\chi$ relation and the observed $\delta-\chi$ relation is well explained by the $d$-spin rotation. As shown by the red dashed line in Fig. 6, we obtained the temperature dependence of the $\delta-\chi$ relation, which increases at low temperature, with the parameters $\mu_{0} H_{\pi-d}=2 \mathrm{~T}$ and $\mathcal{A}=-2.9$, which corresponds to $-4.8 \mathrm{~K}$. In this calculation, the amplitude of the internal field $\mu_{0} H_{\text {int }}=\mu_{0}\left|\boldsymbol{H}_{\pi-d}+\boldsymbol{H}_{d-d}\right|$ increases with decreasing temperature as $3.3,4.6,5.3 \mathrm{~T}$ at $16,8,4 \mathrm{~K}$, respectively. The obtained net internal field is nearly consistent with the estimated value from the temperature dependence of $\chi$ with the $\pi$-ordering model, $4.7 \mathrm{~T}$ without its temperature dependence. In our estimation, $\boldsymbol{H}_{\text {int }}$ shows a large temperature dependence around $T_{\mathrm{AF}} / 2 \sim 8 \mathrm{~K}$ due to the increase of $\boldsymbol{H}_{d-d}$. The calculation for the temperature dependence of the local magnetization for both of the $\pi$ and $d$-spin sites has been conducted considering the $\pi-d$ and $d-d$ interactions [13]. This calculation suggests that the magnetization of $d$-spins grows rapidly at around $T_{\mathrm{AF}} / 2$ when the $d-d$ interaction is as large as the $\pi-d$ interaction and it can well explain our result. Although the temperature dependence of $\boldsymbol{H}_{d-d}$ modifies the behavior of $\chi$ in the $\pi$-ordering model, the differences are not significant. Based on the above discussion, the $d$ - $d$ interaction is expected to assist the stabilization of the AF ordering of the $\pi$-spin system.

In a recent report on $\lambda$-(BETS) $)_{2} \mathrm{Fe}_{x} \mathrm{Ga}_{1-x} \mathrm{Cl}_{4}$, it was pointed out that $H_{\pi-d}$ depends on the Fe spin density $x$, where $H_{\pi-d}$ is roughly proportional to $x$ for $x>0.4$ [14]. This experimental result was noted as a puzzling problem because there is no reason for the high Fe spin density $x$ to increase $H_{\pi-d}$ due to the saturation magnetization of the AF-ordered $\pi$-spin system. However, in addition to the contribution from $H_{\pi-d}$, the internal field on the $d$-spin site also contains contributions from $H_{d-d}$. Therefore, we can solve this problem by considering the 
contribution due to $H_{d-d}$. Since the number of neighboring $d$ spins is reduced with decreasing $x, H_{d-d}$ is effectively reduced as well. Accordingly, the effects of $\lambda$-(BETS) ${ }_{2} \mathrm{Fe}_{x} \mathrm{Ga}_{1-x} \mathrm{Cl}_{4}$ and $\lambda$-(STF $)_{2} \mathrm{FeCl}_{4}$ are well explained in the framework of the $\pi$-ordering model extended by the inclusion of the $d-d$ exchange coupling. We find that all of the $\lambda$-type salts can be placed on the universal phase diagram and can be explained within the same framework considering the $\pi-d$ and $d-d$ interactions.

\section{CONCLUSION}

We have synthesized single crystals of $\lambda$-(STF $)_{2} \mathrm{FeCl}_{4}$ and find that it shows an insulating behavior at ambient pressure like $\mathrm{GaCl}_{4}$ salt from the resistivity measurement. Then, to investigate the $\pi-d$ interaction system, we measured the static susceptibility and ${ }^{1} \mathrm{H}-\mathrm{NMR}$. From the temperature dependence of $T_{1}^{-1}$ observed in ${ }^{1} \mathrm{H}-\mathrm{NMR}$, we determined that $T_{\mathrm{AF}}=16 \mathrm{~K}$ at ambient pressure. The temperature dependence of the spin susceptibility, which shows a broad hump structure without any anomaly at $T_{\mathrm{AF}}$, suggests that the AF transition at $16 \mathrm{~K}$ is due to the AF long-range ordering in the $\pi$-spin system. We find a non-negligible effect of the $d$ - $d$ AF interaction on the effective field, which is comparable to that of the $\pi-d$ interaction, from the relation between the ${ }^{1} \mathrm{H}-\mathrm{NMR}$ splitting associated with the AF and the static susceptibility, which is sensitive to the rotation of the local $d$-spin moment. The AF phases in $\lambda$-type salts can be universally explained by the $\pi$-ordering model extended with the large contribution of the $d$ - $d$ AF exchange field.

\section{ACKNOWLEDGMENTS}

We would like to thank Prof. S. Noro for the x-ray diffraction analysis. This study was partly supported by Hokkaido University, Global Facility Center (GFC), Advanced Physical Property Open Unit (APPOU), funded by Ministry of Education, Culture, Sport, Science and Technology under "Support Program for Implementation of New Equipment Sharing System."
[1] S. Uji, H. Shinagawa, T. Terashima, T. Yakabe, Y. Terai, M. Tokumoto, A. Kobayashi, H. Tanaka, and H. Kobayashi, Nature (London) 410, 908 (2001).

[2] L. Balicas, J. S. Brooks, K. Storr, S. Uji, M. Tokumoto, H. Tanaka, H. Kobayashi, A. Kobayashi, V. Barzykin, and L. P. Gor'kov, Phys. Rev. Lett. 87, 067002 (2001).

[3] V. Jaccarino and M. Peter, Phys. Rev. Lett. 9, 290 (1962).

[4] H. Akutsu, E. Arai, H. Kobayashi, H. Tanaka, A. Kobayashi, and P. Cassoux, J. Am. Chem. Soc. 119, 12681 (1997).

[5] L. Brossard, R. Clerac, C. Coulon, M. Tokumoto, T. Ziman, D. K. Petrov, V. N. Laukhin, M. J. Naughton, A. Audouard, F. Goze, A. Kobayashi, H. Kobayashi, and P. Cassoux, Eur. Phys. J. B 1, 439 (1998).

[6] M. Tokumoto, T. Naito, H. Kobayashi, A. Kobayashi, V. N. Laukhin, L. Brossard, and P. Cassoux, Synth. Met. 86, 2161 (1997).

[7] H. Akiba, S. Nakano, Y. Nishio, K. Kajita, B. Zhou, A. Kobayashi, and H. Kobayashi, J. Phys. Soc. Jpn. 78, 033601 (2009).
[8] H. Akiba, K. Nobori, K. Shimada, Y. Nishio, K. Kajita, B. Zhou, A. Kobayashi, and H. Kobayashi, J. Phys. Soc. Jpn. 80, 063601 (2011).

[9] T. Sasaki, H. Uozaki, S. Endo, and N. Toyota, Synth. Met. 120, 759 (2001).

[10] J. C. Waerenborgh, S. Rabaca, M. Almeida, E. B. Lopes, A. Kobayashi, B. Zhou, and J. S. Brooks, Phys. Rev. B 81, 060413 (2010).

[11] T. Minamidate, Y. Oka, H. Shindo, T. Yamazaki, N. Matsunaga, K. Nomura, and A. Kawamoto, J. Phys. Soc. Jpn. 84, 063704 (2015).

[12] H. Mori, H. Suzuki, T. Okano, H. Moriyama, Y. Nishio, K. Kajita, M. Kodani, K. Takimiya, and T. Otsubo, J. Solid State Chem. 168, 626 (2002).

[13] H. Shimahara and K. Ito, J. Phys. Soc. Jpn. 83, 114702 (2014).

[14] H. Akiba, H. Sugawara, K. Nobori, K. Shimada, N. Tajima, Y. Nishio, K. Kajita, B. Zhou, A. Kobayashi, and H. Kobayashi, J. Phys. Soc. Jpn. 81, 053601 (2012). 